\title{
Giant Pelvic Adenoma Originating from Ectopic Prostatic Tissue
}

\author{
Xiao-Qing Lu, Hai-Ping Hu, Zhi-Cheng Cong and Jian-Di Yu
}

\begin{abstract}
Ectopic prostatic tissue detected outside the genitourinary system has been rarely reported. A case with a giant pelvic adenoma that originated from ectopic prostatic tissue is presented. A 71-year male was detected with lower abdominal mass for eight months and recurrent acute urinary retention for one week. This patient already had mild lower urinary tract symptoms for three years. The physical examination, laboratory tests, ultrasonography, and MRI of this patient were analysed, and the pathological diagnosis was ectopic prostatic adenoma. The suprapubic incision for pelvic exploration and tumorectomy was chosen. The recognition and awareness of this unusual lesion is important, in order not to confuse this particular lesion with other pelvic tumors.
\end{abstract}

Key Words: Giant pelvic adenoma, Ectopic prostatic tissue, Prostatic adenoma.

\section{INTRODUCTION}

Ectopic prostatic tissue may occasionally be found in the posterior urethra or bladder wall, in accordance with embryonic development.1,2 However, to date, ectopic prostatic tissues detected outside the genitourinary tract in the pelvic cavity is very rarely reported, not to mention giant mass lesions. ${ }^{3}$ Since imaging studies are inconclusive with a variety of differential diagnoses, a giant pelvic mass often requires a well-planned open surgery and intraoperative fast frozen section analysis as a diagnostic and therapeutic approach.

\section{CASE REPORT}

A 71-year Chinese male was admitted to the hospital due to detection of a lower abdominal mass for eight months, and recurrent acute urinary retention for one week. This patient already had mild lower urinary tract symptoms, such as frequency, nocturia (2-3 times), and dysuria (for three years). He had taken medicines (tamsulosin etc.) irregularly for the treatment of benign prostatic hypertrophy, without any auxiliary examination at a local hospital. This patient suffered from first episode of acute urinary retention approximately eight months ago, and a lower abdominal mass was found by prostatic ultrasonography for the first time. However, this patient refused surgical treatment for fear of high medical risks at that time. After receiving an indwelling catheter for one week, this patient succeeded for avoiding trial with mild urinary symptoms, as previously. In the patient's past medical history, he only had mild hypertension, which was well-controlled for five years.

Department of Surgical Urology, Zhejiang Hospital,

Hangzhou 310013, China

Correspondence: Dr. Jian-Di Yu, Department of Surgical

Urology, Zhejiang Hospital, No. 12, Lingyin Road, Hangzhou

310013, China

E-mail: yujd.pn@gmail.com

Received: May 15, 2018; Accepted: September 05, 2018
On physical examination, the blunt round upper margin of the solid mass could be touched in the right lower quadrant of the abdomen, while on digital rectal examination, Grade II prostatic enlargement was detected, and a giant pelvic mass was attached to it. The laboratory study revealed elevated PSA $(44.48 \mathrm{ng} / \mathrm{ml}$, normal: 0-4 ng/ml, CA19-9 (48.91 U/ml, normal: 0-39.00 $\mathrm{U} / \mathrm{ml})$, and CA242 (21.42 IU/ml, normal: 0-15.00 IU/ml).

The previous study with ultrasonography showed a heterogeneous mass located under the bladder, which was approximately $12.0 \times 10.6 \mathrm{~cm}$ in size, but the renal collecting system was not affected. On computed tomography (CT) study, the tumor was wellcircumscribed and heterogenous, and no sign of lymphadenopathy or osseous lesions were noted (Figure 1). MRI revealed a large mass lesion (approximately $12.0 \times 10.7 \times 9.5 \mathrm{~cm}$ ) in the pelvic cavity surrounded by a capsule-like structure, which was posterior to the cranially displacing bladder, and closely correlated to the left lobe of the prostate and seminal vesicle (Figure 2). The tissue had a weak signal only on T2 scans (Figure $2 \mathrm{C}$ ). A tentative diagnosis of pelvic low malignant potential sarcoma was made, and the possibility of adenoma with prostate or seminal vesicle origin could not be ruled out.

A suprapubic incision for pelvic exploration and tumorectomy was chosen. Intraoperative frozen section analysis suggested the pelvic mass lesion as an adenoma of unknown origin with moderate dysplasia. Hence, complete tumor resection and right ureteral re-implantation (in order to avoid rupture of right ureter and ureterovesical junction) were performed under general anesthesia (Figure 3). During the procedure, it was found that the encapsulated tumor mass, posterior to the bladder, occupied the whole pelvic space, with its pedicle-like upper part located slightly left to the seminal vesicle and prostate. The pathological study suggested no signs of infiltrating growth and no carcinomatous 


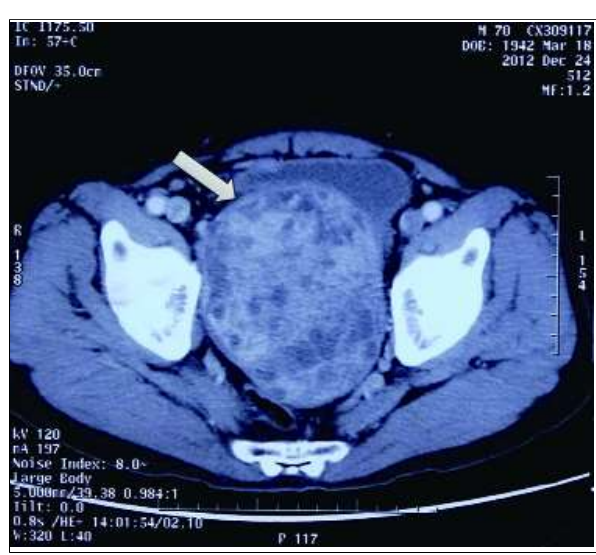

Figure 1: Computed tomography (CT) scan showing the $12 \times 11 \mathrm{~cm}$ pelvic mass. The mass adjacent to the prostate was well-circumscribed with a surrounding clear boundary, and was slightly enhanced on contrast scan.

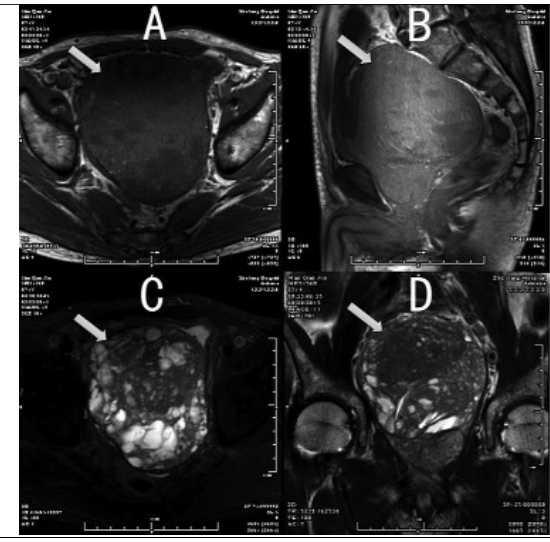

Figure 2: Magnetic resonance imaging (MRI): (A) the T1-weighted SE sequence of the MRI; (B) the sagittal plane of the pelvic imaging; (C) the T2-weighted SE sequence of the MRI; (D) the coronal plane of the pelvic imaging. The arrows show the tumor mass. MRI reveals a moderate hypersignal similar to benign prostatic hyperplasia on the T2-weighted SE sequence.

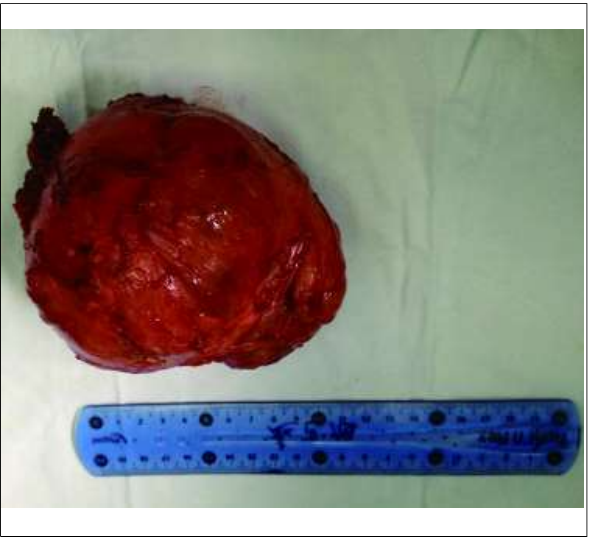

Figure 3: The specimen of the giant adenoma was obtained from the tumorectomy. It was a large mass lesion of approximately $12.0 \times 10.6 \times 9.0 \mathrm{~cm}$.

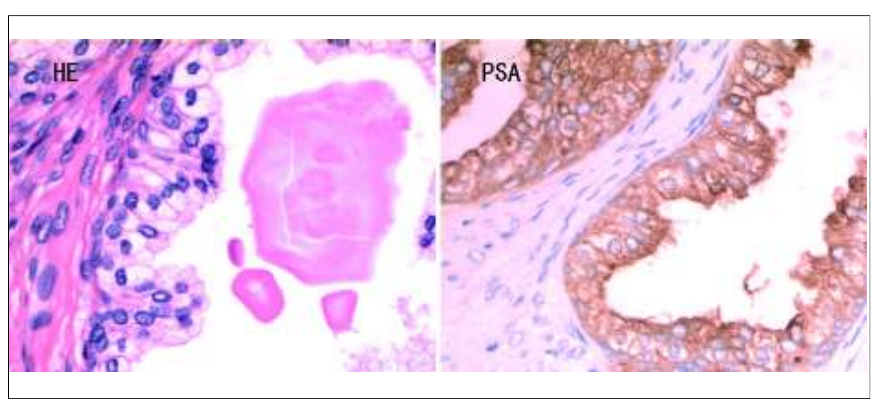

Figure 4: Hematoxylin-eosin $(H \& E)$ staining (magnification, $\times 400$ ) and immunohistochemistry results of the prostate specific antigen (PSA) detection. (A) Glands with light-pink secretions in the glandular lumens were formed by double layered epithelial cells, which had a flat and columnar shape, and a pale cytoplasm, but presented no signs of mitoses and prominent nucleoli. (B) The immunohistochemistry of the tumor cells were positive for PSA.

areas were present in the whole specimen, and the tumor had an intact fibrous capsule surrounding hyperplastic glands and mesenchymal tissues, with some glands having a cystic appearance. The glands were lined by double-layered epithelial cells and exhibited no signs of mitoses and prominent nucleoli (Figure 4A). Immunohistochemically, these tumor cells were positive for PSA (Figure 4B), 34BE12, CD10 and cytokeratin (CK), but negative for CK7, CK20, CEA, P63 and $\mathrm{P} 504 \mathrm{~S}$. The pathological diagnosis was ectopic prostatic adenoma.

\section{DISCUSSION}

Ectopic prostatic tissue, which is an uncommon aberration, may be incidentally found in the urinary tract, such as the posterior urethra and bladder. However, occasional cases may be found in testis, epididymis, penis and seminal vesicle, and even in the anal canal.4-6 This suggests that the embryonic prostatic tissue can migrate and was be found in ectopic sites. In the present case, authors thought that this ectopic prostatic lesion may have originated from seminal vesicle due to the anatomic position of the mass.

Ectopic prostatic tissue outside the urinary tract seldom causes low urinary tract symptoms, unless the lesion has reached large size to cause compression in the tract. Hence, it remains difficult to detect this at earlier stage without an imaging study. When pelvic giant mass lesions develop and are found, it is crucial to obtain a clear working diagnosis of the tumor entity prior to planning the therapeutic procedure. A single imaging test (ultrasound, CT, or MRI) is often not sufficient, and it cannot reveal the exact anatomic relations, although MRI appears to be the best imaging modality to determine the nature of the pelvic mass. For the present case, MRI revealed a moderate hypersignal similar to benign prostatic hyperplasia on the T2-weighted SE sequence, and a low-signal ring was attributed to the capsular fibrous tissue at pathology. In addition, the mass was in contact with the left seminal vesicle and base of the prostate and beneath the bladder. This may suggest its possible origin from ectopically situated prostatic tissue in the seminal vesicle.

Tumors in adult pelvis of male patients may have diverse origins, such as teratoma, lipoma, fibroids, lymphoma, adenocarcinoma, sarcoma, etc. Hence, it is often difficult to arrive at a precise diagnosis before surgery. Since the present differential diagnoses included sarcoma of the pelvis, the investigators deliberately preferred intraoperational frozen section to preoperative transrectal biopsy due to fear of potential tumor spreading. During the surgical procedure, prophylactic isolation and reestablishment of the pelvic organs may have to be taken for the complete resection of such giant pelvic tumor.

In conclusion, the recognition and awareness of this unusual lesion is important, in order not to confuse this particular lesion with other pelvic tumors. 


\section{REFERENCES}

1. Halat S, Eble JN, Grignon DJ, Lacy S, Montironi R, MacLennan GT, et al. Ectopic prostatic tissue: histogenesis and histopathological characteristics. Histopathology 2011; 58:750-8.

2. Bellezza G, Sidoni A, Cavaliere A. Ectopic prostatic tissue in the bladder. Int J Urol 2005; 12:1066-8.

3. Ito $\mathrm{H}$, Fuse $\mathrm{H}$, Hirano $\mathrm{S}$, Masuda $\mathrm{S}$. Ectopic prostatic tissue outside the urinary tract: A case report. Int $J$ Urol 1998; 5:391-2.
4. Salem CE, Gibbs PM, Highshaw RA, Reuter VE and Cote RJ. Benign ectopic prostatic tissue involving the seminal vesicle in a patient with prostate cancer: recognition and implications for staging. Urology 1996; 48:490-3.

5. Tekin K, Sungurtekin U, Aytekin FO, Calli N, Erdem E, Ozden A, et al. Ectopic prostatic tissue of the anal canal presenting with rectal bleeding: report of a case. Dis Colon Rectum 2002; 45:979-80.

6. Van de Griendt EJ, Vijverberg PL, Elbers JR. Ectopic prostatic tissue in the pendulous urethra. Br J Urol 1998; 81:326.

.......... 